TRANSACTIONS OF THE

AMERICAN MATHEMATICAL SOCIETY

Volume 354, Number 12, Pages 4871-4892

S 0002-9947(02)03087-8

Article electronically published on July 16, 2002

\title{
HILBERT TRANSFORMS AND MAXIMAL FUNCTIONS ALONG VARIABLE FLAT CURVES
}

\author{
JONATHAN M. BENNETT
}

\begin{abstract}
We study certain Hilbert transforms and maximal functions along variable flat curves in the plane. We obtain their $L^{2}\left(\mathbb{R}^{2}\right)$ boundedness by considering the oscillatory singular integrals which arise from an application of a partial Fourier transform.
\end{abstract}

\section{Introduction}

This paper is devoted to the study of the Hilbert transform $\mathcal{H}$ given by

$$
\mathcal{H} f\left(x_{1}, x_{2}\right)=p \cdot v \cdot \int_{-\infty}^{\infty} f\left(x_{1}-t, x_{2}-P\left(x_{1}\right) \gamma(t)\right) \frac{d t}{t}
$$

and the maximal function $\mathcal{M}$ given by

$$
\mathcal{M} f\left(x_{1}, x_{2}\right)=\sup _{h>0} \frac{1}{h}\left|\int_{0}^{h} f\left(x_{1}-t, x_{2}-P\left(x_{1}\right) \gamma(t)\right) d t\right|,
$$

for $P$ a real-valued polynomial, and $\gamma: \mathbb{R} \rightarrow \mathbb{R}$ satisfying certain growth conditions. What is of prime interest to us is that these conditions will not exclude those $\gamma$ that vanish to infinite order at the origin. For example, $\gamma$ may behave like $\exp \left(-t^{-2}\right)$ for small $t$.

Theorem 1. Let $P: \mathbb{R} \rightarrow \mathbb{R}$ be a polynomial, and let $\gamma \in C^{3}(\mathbb{R})$ be either odd or even, convex on $(0, \infty)$, and satisfy

(i) $\gamma(0)=\gamma^{\prime}(0)=0$,

(ii) $\lambda(t)=t \gamma^{\prime \prime}(t) / \gamma^{\prime}(t)$ is decreasing and bounded below on $(0, \infty)$.

Then the Hilbert transform $\mathcal{H}$ is bounded on $L^{2}\left(\mathbb{R}^{2}\right)$ with a bound that can be taken to be independent of the coefficients of $P$.

Theorem 2. If $P$ and $\gamma$ satisfy the conditions of Theorem 1] then the maximal function $\mathcal{M}$ is bounded on $L^{2}\left(\mathbb{R}^{2}\right)$ with a bound that can be taken to be independent of the coefficients of $P$.

Most of the work that has been done in this area, with regard to flat curves, has been in the translation-invariant setting; see, for example, [6] and [2]. Seeger [10] and Carbery and Pérez [3] have obtained results for translation-invariant families of flat curves that do, however, take one out of the realm of translation-invariant operators. Another approach to the study of non-translation-invariant operators

Received by the editors May 4, 1999.

2000 Mathematics Subject Classification. Primary 44A12, 42 B20.

Partially supported by EPSRC Grant GR/L10024. 
has been to view them as translation invariant on some Lie group. In particular, Carbery, Wainger and Wright considered certain translation-invariant operators on the Heisenberg group; see [4. When $P\left(x_{1}\right)=x_{1}$, Theorems 1 and 2 are contained in the work of Carbery, Wainger and Wright [5], which was motivated by their previous work on the Heisenberg group. Recent work of Kim 7], again on the Heisenberg group, implies some sharp results on $L^{2}$ in this case. The operators (1) and (2) that we consider can, in principle, be interpreted in a similar way, although we do not explicitly appeal to any translation-invariant structure here.

An amusing feature of the proof of Theorems 1 and 2 is that if we did not look for independence of the coefficients, we would be unable to conclude that the operators were bounded. This is because our proof goes by induction, and the independence claim in the inductive hypothesis is crucial. A similar phenomenon arose in work of Ricci and Stein 9 .

We would like to thank A. Carbery for his helpful thoughts and advice during the development of this work, as part of the author's Ph.D. thesis [1].

\section{Preliminaries}

We begin by recalling some simple properties of the curves $\gamma$.

Lemma 3. $\gamma^{\prime}$ is doubling; i.e., there exists $C<\infty$ for which

$$
\gamma^{\prime}(C t) \geq 2 \gamma^{\prime}(t) \text { for all } t>0 .
$$

Proof. If $\lambda_{0}=\inf _{t>0} \lambda(t)$ and $C=e^{\frac{2}{\lambda_{0}}}$, then

$$
\gamma^{\prime}(C t)=\int_{0}^{C t} \gamma^{\prime \prime}(s) d s \geq \int_{t}^{C t} \gamma^{\prime \prime}(s) d s \geq \lambda_{0} \int_{t}^{C t} \frac{\gamma^{\prime}(s)}{s} d s \geq \lambda_{0} \gamma^{\prime}(t) \log C=2 \gamma^{\prime}(t),
$$

for all $t>0$.

Lemma 4. If $g(s, t)=\frac{\gamma^{\prime}(s)-\gamma^{\prime}(t)}{\gamma(s)-\gamma(t)}$, then

Proof.

$$
\frac{\partial g}{\partial s} \leq 0 \text { for } s, t \geq 0, s \neq t .
$$

$$
\begin{aligned}
\frac{\partial g}{\partial s} & =\frac{(\gamma(s)-\gamma(t)) \gamma^{\prime \prime}(s)-\left(\gamma^{\prime}(s)-\gamma^{\prime}(t)\right) \gamma^{\prime}(s)}{(\gamma(s)-\gamma(t))^{2}} \\
& =\frac{\gamma^{\prime}(s)}{\gamma(s)-\gamma(t)}\left(\frac{\gamma^{\prime \prime}(s)}{\gamma^{\prime}(s)}-\frac{\gamma^{\prime}(s)-\gamma^{\prime}(t)}{\gamma(s)-\gamma(t)}\right) \\
& =\frac{\gamma^{\prime}(s)}{\gamma(s)-\gamma(t)}\left(\frac{\gamma^{\prime \prime}(s)}{\gamma^{\prime}(s)}-\frac{\gamma^{\prime \prime}(\theta)}{\gamma^{\prime}(\theta)}\right),
\end{aligned}
$$

for some $\theta$ between $s$ and $t$, by the Generalised Mean Value Theorem. Since $\frac{\gamma^{\prime \prime}}{\gamma^{\prime}}$ is decreasing, $\frac{\partial g}{\partial s} \leq 0$.

Lemma 5. There exists $c>0$ for which

$$
\gamma(s)-\gamma(t) \geq c(s-t) \gamma(s)
$$

and

$$
\gamma^{\prime}(s)-\gamma^{\prime}(t) \geq c(s-t) \gamma^{\prime}(s),
$$

for all $1 \leq t \leq s \leq 2$. 
Proof. We will prove (5); (4) is similar. We may suppose that $\gamma^{\prime}(t)>\frac{1}{2} \gamma^{\prime}(s)$ since, on the other hand,

$$
\gamma^{\prime}(s)-\gamma^{\prime}(t) \geq \frac{1}{2} \gamma^{\prime}(s) \geq \frac{1}{2}(s-t) \gamma^{\prime}(s)
$$

for $1 \leq t \leq s \leq 2$.

$$
\text { If } \gamma^{\prime}(t)>\frac{1}{2} \gamma^{\prime}(s) \text {, then }
$$

$$
\gamma^{\prime}(s)-\gamma^{\prime}(t)=\int_{t}^{s} \gamma^{\prime \prime}(x) d x=\int_{t}^{s} \lambda(x) \frac{\gamma^{\prime}(x)}{x} d x \geq \frac{\lambda_{0}}{2}(s-t) \gamma^{\prime}(t) \geq \frac{\lambda_{0}}{4}(s-t) \gamma^{\prime}(s),
$$

for $1 \leq t \leq s \leq 2$.

In what follows we shall need the following well-known lemma, which is a consequence of the Mean Value Theorem.

Lemma 6. If $P$ is a real monic polynomial of one variable, and of degree $n$, then there is a constant $C$ which depends only on $n$ for which

$$
|\{x \in \mathbb{R}:|P(x)| \leq \delta\}| \leq C \delta^{\frac{1}{n}}
$$

for all $\delta>0$.

Lemma 7. Suppose that $T$ is an $L^{p}\left(\mathbb{R}^{n}\right)$ bounded operator for some $1 \leq p \leq \infty$, and has integral kernel $K(x, y)$. If $\phi \in C_{c}^{\infty}\left(\mathbb{R}^{n}\right)$, then the operator $T_{\phi}$ with integral kernel $K(x, y) \phi(x-y)$ is bounded on $L^{p}\left(\mathbb{R}^{n}\right)$, and $\left\|T_{\phi}\right\|_{p-p} \leq\|\widehat{\phi}\|_{1}\|T\|_{p-p}$.

Proof. By writing $\phi$ as the inverse Fourier transform of $\widehat{\phi}$, we have

$$
\begin{aligned}
T_{\phi} f(x) & =\int_{\mathbb{R}^{n}} \widehat{\phi}(\xi) \int_{\mathbb{R}^{n}} e^{-2 \pi i \xi \cdot(x-y)} K(x, y) f(y) d y d \xi \\
& =\int_{\mathbb{R}^{n}} \widehat{\phi}(\xi) e^{2 \pi i \xi \cdot x} \int_{\mathbb{R}^{n}} K(x, y) e^{-2 \pi i \xi \cdot y} f(y) d y d \xi \\
& =\int_{\mathbb{R}^{n}} \widehat{\phi}(\xi) e^{-2 \pi i \xi \cdot x} T f_{\xi}(x) d \xi,
\end{aligned}
$$

where $f_{\xi}(y)=e^{2 \pi i \xi \cdot y} f(y)$. Since $\left\|f_{\xi}\right\|_{p}=\|f\|_{p}$ for all $\xi \in \mathbb{R}^{n}$, the conclusion of Lemma 7 follows by Minkowski's inequality for integrals.

Before we begin the proof of Theorem [1, we need to introduce the notion of the "Minkowski content" of a subset of $\mathbb{R}^{2}$. If $0 \leq d \leq 2$, a set $E \subset \mathbb{R}^{2}$ is said to have $d$-dimensional Minkowski content bounded above by $C$ if

$$
\left|E_{\delta}\right| \leq C \delta^{2-d}
$$

for $\delta \leq 1$, where

$$
E_{\delta}=\left\{x \in \mathbb{R}^{2}: \operatorname{dist}(x, E)<\delta\right\} .
$$

Example. Suppose $\varphi: \mathbb{R} \rightarrow \mathbb{R}$ is a monotone function and $\Gamma=\{(x, \varphi(x)): x \in \mathbb{R}\}$. If $\square$ is the interior of the unit square in $\mathbb{R}^{2}$, then the 1 -dimensional Minkowski content of the set $\Gamma \cap \square$ is bounded above by 9 . In what follows, our conclusions concerning Minkowski content arise in this way. 


\section{The Proof of Theorem 1}

The proof of Theorem 1 will proceed by induction on the degree of the polynomial.

By an application of a partial Fourier transform 1 the $L^{2}\left(\mathbb{R}^{2}\right)$ boundedness of $\mathcal{H}$, given by (1), is equivalent to the $L^{2}(\mathbb{R})$ boundedness of the oscillatory singular integrals

$$
T f(x)=p \cdot v \cdot \int_{-\infty}^{\infty} \frac{e^{i P(x) \gamma(x-y)}}{x-y} f(y) d y
$$

uniformly in the coefficients of $P$. We will, however, show that these oscillatory singular integrals are uniformly bounded on $L^{p}(\mathbb{R})$ for $1<p<\infty$.

The inductive hypothesis. Suppose $T$, given by (6) , is bounded on $L^{p}(\mathbb{R})$ for all polynomials $P$ of degree less than $n$, with a bound that is independent of the coefficients of $P$, for $1<p<\infty$.

The case $n=0$. When $n=0$, the class of operators is reduced to

$$
T f(x)=\int_{-\infty}^{\infty} \frac{e^{i \lambda \gamma(x-y)}}{x-y} f(y) d y
$$

for $\lambda \in \mathbb{R}$. By the use of a partial Fourier transform, uniform (in $\lambda$ ) $L^{p}(\mathbb{R})$ boundedness of $T$ is a consequence of the $L^{p}\left(\mathbb{R}^{2}\right)$ boundedness of the corresponding $\mathcal{H}$, which is given by

$$
\mathcal{H} f(x)=\int_{-\infty}^{\infty} f\left(x_{1}-t, x_{2}-\gamma(t)\right) \frac{d t}{t} .
$$

The proof of this can be found in $[6]$.

- Let $P$ be a polynomial of degree $n$. Firstly, we shall observe that it is enough to consider $P$ monic, and $\gamma$ satisfying $\gamma(1)=1$. Suppose $s$ is the coefficient of $x^{n}$ in $P$, and that $\omega$ satisfies $s \omega^{n} \gamma(\omega)=1$. Now,

$$
T f(\omega x)=\int_{-\infty}^{\infty} \frac{e^{i P(\omega x) \gamma(\omega(x-y))}}{x-y} f(\omega y) d y=\int_{-\infty}^{\infty} \frac{e^{i \widetilde{P}(x) \widetilde{\gamma}(x-y)}}{x-y} f(\omega y) d y,
$$

where $\widetilde{P}(x)=\gamma(\omega) P(\omega x)$ and $\widetilde{\gamma}(x)=\gamma(\omega x) / \gamma(\omega)$. We now simply observe that $\widetilde{P}$ is monic, $\widetilde{\gamma}$ satisfies the conditions of Theorem 1 and $\widetilde{\gamma}(1)=1$. Since the $L^{p}(\mathbb{R})$ operator norm of $T$ is equal to that of

$$
\widetilde{T} f(x)=\int_{-\infty}^{\infty} \frac{e^{i \widetilde{P}(x)} \widetilde{\gamma}(x-y)}{x-y} f(y) d y
$$

our claim follows. In what follows $P$ will be monic, and $\gamma$ will be "normalised" in the sense that $\gamma(1)=1$.

We now decompose

$$
T=T^{(1)}+\sum_{k \geq 0} T_{k}
$$

where

$$
T^{(1)} f(x)=\int_{|x-y| \leq 1} \frac{e^{i P(x) \gamma(x-y)}}{x-y} f(y) d y
$$

\footnotetext{
${ }^{1}$ See Phong and Stein [8] for background and discussion.
} 
and

$$
T_{k} f(x)=\int_{2^{k} \leq|x-y| \leq 2^{k+1}} \frac{e^{i P(x) \gamma(x-y)}}{x-y} f(y) d y
$$

THE LOCAL PART $T^{(1)}$

Since the integral defining $T^{(1)}$ is restricted to $|x-y| \leq 1$, it suffices to consider $T^{(1)}$ acting on functions supported in balls of radius 1 . Suppose $f \in L^{p}(\mathbb{R})$ is such a function, and has centre $b$. Let $Q_{b}(x)=P(x)-(x-b)^{n}$. Since $Q_{b}$ is a polynomial of degree less than $n$, by the inductive hypothesis,

$$
S_{b} f(x)=\int_{-\infty}^{\infty} \frac{e^{i Q_{b}(x) \gamma(x-y)}}{x-y} f(y) d y
$$

is bounded on $L^{p}(\mathbb{R})$ with bound independent of $b$ and the coefficients of $P$. Let $\phi \in C_{c}^{\infty}(\mathbb{R})$ be such that $\phi(t)=1$ when $|t| \leq 1$. By Lemma 7

$$
S_{b, \phi} f(x)=\int_{-\infty}^{\infty} \frac{e^{i Q_{b}(x) \gamma(x-y)}}{x-y} \phi(x-y) f(y) d y
$$

is also bounded on $L^{p}(\mathbb{R})$ with bound independent of $b$ and the coefficients of $P$.

We now define the operator $S_{b}^{l}$ by

$$
S_{b}^{l} f(x)=\int_{|x-y| \leq 1} \frac{e^{i Q_{b}(x) \gamma(x-y)}}{x-y} f(y) d y .
$$

On observing that

$$
\left|S_{b, \phi} f(x)-S_{b}^{l} f(x)\right| \leq|\phi| *|f|(x),
$$

we conclude that $S_{b}^{l}$ is bounded on $L^{p}(\mathbb{R})$ with bound independent of $b$ and the coefficients of $P$.

Now,

$$
\begin{aligned}
\left|T^{(1)} f(x)-S_{b}^{l} f(x)\right|= & \left|\int_{|x-y| \leq 1} \frac{e^{i P(x) \gamma(x-y)}-e^{i Q_{b}(x) \gamma(x-y)}}{x-y} f(y) d y\right| \\
\leq & |x-b|^{n} \int_{|x-y| \leq 1}\left|\frac{\gamma(x-y)}{x-y}\right||f(y)| d y \\
\leq & 2^{n} \int_{|x-y| \leq 1}|f(y)| d y \\
& (\text { since } \gamma \text { is convex, } \gamma(0)=0, \text { and } \gamma(1)=1) \\
= & 2^{n} A f(x),
\end{aligned}
$$

where $A$ is the averaging operator given by

$$
A f(x)=\int_{|x-y| \leq 1}|f(y)| d y .
$$

Since $A$ is trivially bounded on $L^{p}(\mathbb{R}), T^{(1)}$ is bounded on $L^{p}(\mathbb{R})$ for $1<p<\infty$, with a bound that is independent of the coefficients of $P$. 
Define the operator $\widetilde{T}_{k}$ by

$$
\widetilde{T}_{k} f(x)=T_{k}\left(f\left(2^{-k} \cdot\right)\right)\left(2^{k} x\right)
$$

i.e.,

$$
\widetilde{T}_{k} f(x)=\int_{1 \leq|x-y| \leq 2} \frac{e^{i 2^{n k} \gamma\left(2^{k}\right) \widetilde{P}_{k}(x) \widetilde{\gamma}_{k}(x-y)}}{x-y} f(y) d y,
$$

where $\widetilde{P}_{k}(x)=2^{-n k} P\left(2^{k} x\right)$, and $\widetilde{\gamma}_{k}(x)=\gamma\left(2^{k} x\right) / \gamma\left(2^{k}\right)$. We should remark that this type of rescaling preserves the operator norm.

Since $\gamma$ is either even or odd, we need only consider

$$
\mathbb{T}_{k} f(x)=\int_{1 \leq x-y \leq 2} \frac{e^{i 2^{n k} \gamma\left(2^{k}\right) \widetilde{P}_{k}(x) \widetilde{\gamma}_{k}(x-y)}}{x-y} f(y) d y .
$$

Since we are unable to bound the global part using the oscillation alone, we are forced to define some "bad" sets $E_{k}$, on which we rely entirely on the size of the kernels of the operators $\mathbb{T}_{k}$. Let

$$
E_{k}=\left\{x \in \mathbb{R}:\left(\frac{\widetilde{P}_{k}^{\prime}}{\widetilde{P}_{k}}\right)^{\prime}(x)>0 \text { and }\left|\frac{\widetilde{P}_{k}^{\prime}(x)}{\widetilde{P}_{k}(x)}\right| \geq \frac{\lambda_{0}}{4}\right\},
$$

where

$$
\lambda_{0}=\inf _{t>0} \lambda(t)
$$

We define the "good" part of the operator $\mathbb{T}_{k}$ to be $\mathbb{T}_{k}^{g}$, where

$$
\mathbb{T}_{k}^{g} f(x)=\chi_{E_{k}^{c}}(x) \mathbb{T}_{k} f(x),
$$

and the "bad" part to be

$$
\mathbb{T}_{k}^{b} f(x)=\chi_{E_{k}}(x) \mathbb{T}_{k} f(x)
$$

Lemma 8. Let $P$ be a monic polynomial, and $\gamma$ be as in Theorem 1 , with $\gamma(1)=1$. If, for $\mu>0$,

$$
R_{\mu} f(x)=\chi_{E^{c}}(x) \int_{1 \leq x-y \leq 2} \frac{e^{i \mu P(x) \gamma(x-y)}}{x-y} f(y) d y,
$$

where

$$
E=\left\{x \in \mathbb{R}:\left(\frac{P^{\prime}}{P}\right)^{\prime}(x)>0 \text { and }\left|\frac{P^{\prime}(x)}{P(x)}\right| \geq \frac{\lambda_{0}}{4}\right\},
$$

then there is an $\epsilon>0$ and a constant $A$, independent of the coefficients of $P$, for which

for all $\mu>0$.

$$
\left\|R_{\mu}\right\|_{2-2} \leq A \mu^{-\epsilon}
$$

Lemma 9. For any $\alpha>0$,

$$
\sum_{k \geq 0}\left|E_{k}\right|^{\alpha}
$$

is convergent, with a bound which depends only on $\alpha$ and the degree of the polynomial $P$. 
We first show how Lemmas 8 and 9 imply Theorem 1 ,

By Lemma 8

$$
\left\|\mathbb{T}_{k}^{g}\right\|_{2-2} \leq A\left(2^{n k} \gamma\left(2^{k}\right)\right)^{-\epsilon},
$$

for all $k \geq 0$. Since $\mathbb{T}_{k}^{g}$ is trivially bounded on $L^{1}$ and $L^{\infty}$, uniformly in $k$, by interpolation we have

$$
\sum_{k \geq 0}\left\|\mathbb{T}_{k}^{g}\right\|_{p-p} \leq A \sum_{k \geq 0}\left(2^{n k} \gamma\left(2^{k}\right)\right)^{-\epsilon^{\prime}}<\infty
$$

with bound independent of the coefficients of $P$. Here we have used the facts that $\gamma(1)=1$ and $\gamma$ is increasing.

By interpolating between the trivial estimates,

$$
\left\|\mathbb{T}_{k}^{b} f\right\|_{1} \leq\left|E_{k}\right|\|f\|_{1}
$$

and

$$
\left\|\mathbb{T}_{k}^{b} f\right\|_{\infty} \leq C\|f\|_{\infty}
$$

we obtain

$$
\left\|\mathbb{T}_{k}^{b}\right\|_{p-p} \leq C\left|E_{k}\right|^{\frac{1}{p}}
$$

and so by Lemma 9

$$
\sum_{k \geq 0}\left\|\mathbb{T}_{k}^{b}\right\|_{p-p}<\infty
$$

with bound independent of the coefficients of $P$.

We now turn to the proofs of Lemmas 8 and 9

The proof of Lemma 8 . We will use the fact that $\left\|R_{\mu}\right\|_{2-2}=\left\|R_{\mu}^{*} R_{\mu}\right\|_{2-2}^{1 / 2}$.

Let $L_{\mu}(x, y)$ be the kernel of $R_{\mu}^{*} R_{\mu}$; i.e.,

$$
L_{\mu}(x, y)=\int_{1 \leq z-x, z-y \leq 2 ; z \in E^{c}} \frac{e^{i \mu P(z)(\gamma(z-x)-\gamma(z-y))}}{(z-x)(z-y)} d z .
$$

Let

$$
\psi(x, y, z)=P(z)(\gamma(z-x)-\gamma(z-y))
$$

and

$$
\Delta=\left\{(z, x) \in \mathbb{R}^{2}: 1 \leq z-y \leq z-x \leq 2 ; z \in E^{c}\right\} .
$$

It suffices to consider the kernel

$$
\mathbb{L}_{\mu}(x, y)=\int_{\{z:(z, x) \in \Delta\}} \frac{e^{i \mu \psi(x, y, z)}}{(z-x)(z-y)} d z,
$$

since $L_{\mu}=\mathbb{L}_{\mu}+\mathbb{L}_{\mu}^{*}$.

Since the $L^{\infty}$ operator norm of $R_{\mu}^{*} R_{\mu}$ is bounded uniformly in $\mu$, it is enough, by interpolation, to obtain appropriate decay estimates for its $L^{1}$ operator norm. To this end we seek an estimate of the form

$$
\sup _{y} \int\left|\mathbb{L}_{\mu}(x, y)\right| d x \leq c \mu^{-\epsilon}
$$

for some $\epsilon>0$. 
In what follows we will use the standard notation for partial derivatives where, for a twice differentiable function $f: \mathbb{R}^{n} \rightarrow \mathbb{R}$, one writes $f_{i}$ for $\frac{\partial f}{\partial x_{i}}$, and $f_{i j}$ for $\frac{\partial^{2} f}{\partial x_{i} \partial x_{j}}$.

Let $y \in \mathbb{R}$ be fixed.

Lemma 10. If $\frac{P^{\prime}(z)}{P(z)}<0$, and $z$ is fixed, then there exists at most one value of $x<y$ for which $\psi_{3}(x, y, z)=0$, and at most one for which $\psi_{31}(x, y, z)=0$.

Proof.

$$
\psi_{3}(x, y, z)=P(z)\left(\gamma^{\prime}(z-x)-\gamma^{\prime}(z-y)\right)+P^{\prime}(z)(\gamma(z-x)-\gamma(z-y))
$$

and

$$
\psi_{31}(x, y, z)=-P(z) \gamma^{\prime \prime}(z-x)-P^{\prime}(z) \gamma^{\prime}(z-x)
$$

and so

$$
\frac{\partial}{\partial x}\left(\frac{\psi_{31}(x, y, z)}{\gamma^{\prime}(z-x)}\right)=-P(z) \frac{\partial}{\partial x}\left(\frac{\lambda(z-x)}{z-x}\right)
$$

which is of constant sign for fixed $z$. Hence $\frac{\psi_{31}(x, y, z)}{\gamma^{\prime}(z-x)}$ is a monotone function of $x$, and so has at most one zero. Hence $\psi_{3}$ can have at most two zeros, one of which must be $x=y$.

Lemma 11. For fixed $y$, the zero sets of $\psi_{3}$ and $\psi_{31}$ in $\Delta$ have bounded onedimensional Minkowski content, with bound depending only on the degree of the polynomial $P$. In particular, the bound does not depend on $y$.

Proof. Since $z \notin E$, either

$$
\left|\frac{P^{\prime}(z)}{P(z)}\right| \leq \frac{\lambda_{0}}{4}, \text { or }
$$

$$
\left(\frac{P^{\prime}}{P}\right)^{\prime}(z) \leq 0
$$

If (i), then by the Generalised Mean Value Theorem,

$$
\left|\frac{P^{\prime}(z)(\gamma(z-x)-\gamma(z-y))}{P(z)\left(\gamma^{\prime}(z-x)-\gamma^{\prime}(z-y)\right)}\right|=\left|\frac{P^{\prime}(z)}{P(z)}\right| \frac{\gamma^{\prime}(\theta)}{\gamma^{\prime \prime}(\theta)}=\left|\frac{P^{\prime}(z)}{P(z)}\right| \frac{\theta}{\lambda(\theta)},
$$

for some $\theta \in(1,2)$. Since $\lambda(\theta) \geq \lambda_{0}$, and $\theta \in(1,2)$, ( 8 ) is less than

$$
\left|\frac{P^{\prime}(z)}{P(z)}\right| \frac{2}{\lambda_{0}} \leq \frac{1}{2}
$$

and consequently $\psi_{3}$ has no nontrivial zero as a function of $x$.

In case (ii), if $\psi_{3}$ has a nontrivial zero, say $\alpha_{y}(z)$, as a function of $x$, then it is defined implicitly by

$$
P(z)\left[\gamma^{\prime}\left(z-\alpha_{y}(z)\right)-\gamma^{\prime}(z-y)\right]+P^{\prime}(z)\left[\gamma\left(z-\alpha_{y}(z)\right)-\gamma(z-y)\right]=0
$$

or

$$
g\left(z-\alpha_{y}(z), z-y\right)=-\frac{P^{\prime}(z)}{P(z)}
$$


where $g(s, t)=\frac{\gamma^{\prime}(s)-\gamma^{\prime}(t)}{\gamma(s)-\gamma(t)}$. Since $\psi_{31}$ has at most one zero, and $\psi_{3}$ has at most two zeros (including $x=y$ ) as functions of $x$, then $\psi_{31}\left(\alpha_{y}(z), y, z\right) \neq 0$. This implies, by the Implicit Function Theorem, that $\alpha_{y}$ is defined on an open set $U_{y}$ and is differentiable. Differentiating (9) with respect to $z$ on $U_{y}$ gives

$$
\left(1-\frac{d \alpha_{y}}{d z}\right) \frac{\partial g}{\partial s}\left(z-\alpha_{y}(z), z-y\right)+\frac{\partial g}{\partial t}\left(z-\alpha_{y}(z), z-y\right)=-\left(\frac{P^{\prime}}{P}\right)^{\prime}(z) \geq 0
$$

By Lemma $4 \frac{\partial g}{\partial s} \leq 0$ and $\frac{\partial g}{\partial t} \leq 0$, and so $\frac{d \alpha_{y}}{d z} \geq 1$. Hence $\left\{(z, x) \in \Delta: x=\alpha_{y}(z)\right\}$ has bounded one-dimensional Minkowski content.

We now turn to the zero set of $\psi_{31}$.

If $\psi_{31}$ has a zero, say $\beta_{y}(z)$, as a function of $x$, then it is defined implicitly by

$$
P(z) \gamma^{\prime \prime}\left(z-\beta_{y}(z)\right)+P^{\prime}(z) \gamma^{\prime}\left(z-\beta_{y}(z)\right)=0 .
$$

Clearly $\beta_{y}$ does not depend on $y$; so we simply write $\beta_{y}=\beta$. Since

$$
\frac{\gamma^{\prime \prime}(z-\beta(z))}{\gamma^{\prime}(z-\beta(z))}=-\frac{P^{\prime}(z)}{P(z)}
$$

and $\frac{\gamma^{\prime \prime}}{\gamma^{\prime}}$ is strictly decreasing, $z-\beta(z)$ changes monotonicity exactly when $\frac{P^{\prime}}{P}$ does; i.e., boundedly often. Hence $\{(z, x) \in \Delta: x=z-\beta(z)\}$ has bounded onedimensional Minkowski content. By considering the shear $\Phi: \mathbb{R}^{2} \rightarrow \mathbb{R}^{2}$ given by $\Phi(x, y)=(x, x-y)$ (a global diffeomorphism with Jacobian determinant equal to 1 ), one can deduce that $\{(z, x) \in \Delta: x=\beta(z)\}$ also has bounded one-dimensional Minkowski content.

At this point the following observation is appropriate. Since $\lambda$ is decreasing and bounded below on $(0, \infty), \frac{\gamma^{\prime \prime}(t)}{\gamma^{\prime}(t)} \rightarrow \infty$ as $t \rightarrow 0$, and $\frac{\gamma^{\prime \prime}(t)}{\gamma^{\prime}(t)} \rightarrow 0$ as $t \rightarrow \infty$. Consequently $\frac{\gamma^{\prime \prime}}{\gamma^{\prime}}:(0, \infty) \rightarrow(0, \infty)$ is surjective, and so $\beta$ is defined exactly on the set $\left\{z \in \mathbb{R}: \frac{P^{\prime}(z)}{P(z)}<0\right\}$.

Let

$$
\begin{aligned}
& F_{1}=\{(z, x) \in \Delta: P(z)=0\}, \\
& F_{2}=\left\{(z, x) \in \Delta: P^{\prime}(z)=0\right\}, \\
& F_{3}=\left\{(z, x) \in \Delta: P^{\prime \prime}(z)=0\right\}, \\
& F_{4}=\{(z, x) \in \Delta: x=y\}, \\
& F_{5}=\left\{(z, x) \in \Delta: x=\alpha_{y}(z)\right\}, \\
& F_{6}=\{(z, x) \in \Delta: x=\beta(z)\},
\end{aligned}
$$

and

$$
F_{7}=\partial \Delta
$$

Our aim in what follows is to establish some lower bounds for $\psi_{3}$ on $\Delta$. To do this, we will divide $\Delta$ up into three pieces $\Delta_{1}, \Delta_{2}$, and $\Delta_{3}$, and make estimates of a different type on each. For technical reasons we need to understand the nature of some of the boundaries between these regions; these are given by

$$
F_{8}=\left\{(z, x) \in \Delta: x=\beta_{+}(z)\right\}
$$

and

$$
F_{9}=\left\{(z, x) \in \Delta: x=\beta_{-}(z)\right\}
$$


where $\beta_{+}$and $\beta_{-}$are given by

$$
\frac{1}{2} \frac{\gamma^{\prime \prime}\left(z-\beta_{+}(z)\right)}{\gamma^{\prime}\left(z-\beta_{+}(z)\right)}=-\frac{P^{\prime}(z)}{P(z)}
$$

and

$$
2 \frac{\gamma^{\prime \prime}\left(z-\beta_{-}(z)\right)}{\gamma^{\prime}\left(z-\beta_{-}(z)\right)}=-\frac{P^{\prime}(z)}{P(z)} .
$$

As for $z-\beta(z), z-\beta_{+}(z)$ and $z-\beta_{-}(z)$ change monotonicity boundedly often, and so $F_{8}$ and $F_{9}$ have bounded one-dimensional Minkowski content. As for $\beta$ again, $\beta_{+}$and $\beta_{-}$are defined exactly on $\left\{z \in \mathbb{R}: \frac{P^{\prime}(z)}{P(z)}<0\right\}$.

Let

$$
F=\bigcup_{j=1}^{9} F_{j}
$$

By Lemma 11, $F$ has one-dimensional Minkowski content bounded by a constant depending only on $n=\operatorname{deg}(P)$, i.e., not on $y$ or the coefficients of $P$.

Decompose $\Delta \backslash F$ into a union of Whitney cubes $\left\{B_{l, m}\right\}_{l \geq 0, m \in \mathbb{N}}$ whose sides are parallel to the axes, and for which $\operatorname{diam}\left(B_{l, m}\right)=2^{-l}$. Since $F$ has bounded onedimensional Minkowski content,

$$
\#\left\{B \in\left\{B_{l, m}\right\}: \operatorname{diam}(B)=2^{-l}\right\} \leq C 2^{l} .
$$

Next, we write

$$
\mathbb{L}_{\mu}(x, y)=\int_{\{z:(z, x) \in \Delta\}} e^{i \mu \psi(x, y, z)} \frac{d z}{(z-x)(z-y)}=\sum_{l, m} \mathbb{L}_{l, m}(x, y),
$$

where

$$
\mathbb{L}_{l, m}(x, y)=\int_{\left\{z:(z, x) \in B_{l, m}\right\}} e^{i \mu \psi(x, y, z)} \frac{d z}{(z-x)(z-y)} .
$$

We now claim that it is enough for us to obtain an estimate of the form

$$
\sup _{y} \int\left|\mathbb{L}_{l, m}(x, y)\right| d x \leq C \frac{2^{M l}}{\mu}
$$

for some $M>0$ that is independent of $l$.

Assuming (12), and the trivial estimate,

$$
\sup _{y} \int\left|\mathbb{L}_{l, m}(x, y)\right| d x \leq C 2^{-2 l}
$$

we obtain

$$
\begin{aligned}
\sup _{y} \int\left|\mathbb{L}_{\mu}(x, y)\right| d x & \leq \sum_{l, m} \sup _{y} \int\left|\mathbb{L}_{l, m}(x, y)\right| d x \\
& \leq C \sum_{l, m} \min \left\{2^{-2 l}, 2^{M l} / \mu\right\} \\
& \left.\leq C \sum_{l} \min \left\{2^{-l}, 2^{(M+1) l} / \mu\right\} \quad \text { by (10) }\right) \\
& \leq C \mu^{-\frac{1}{M+2}},
\end{aligned}
$$

as required. From here we will focus on finding an estimate of the form (12). 
Before we integrate by parts in (11), we must establish some lower bounds for $\psi_{3}$ in terms of $\operatorname{dist}((z, x), F)$.

Let

$$
\begin{gathered}
\Delta_{1}=\left\{(z, x) \in \Delta: \frac{P^{\prime}(z)}{P(z)} \geq-\frac{1}{2} \frac{\gamma^{\prime \prime}(z-x)}{\gamma^{\prime}(z-x)}\right\}, \\
\Delta_{2}=\left\{(z, x) \in \Delta: \frac{P^{\prime}(z)}{P(z)}<-2 \frac{\gamma^{\prime \prime}(z-x)}{\gamma^{\prime}(z-x)}\right\}, \\
\Delta_{3}=\left\{(z, x) \in \Delta:-2 \frac{\gamma^{\prime \prime}(z-x)}{\gamma^{\prime}(z-x)} \leq \frac{P^{\prime}(z)}{P(z)} \leq-\frac{1}{2} \frac{\gamma^{\prime \prime}(z-x)}{\gamma^{\prime}(z-x)}\right\} .
\end{gathered}
$$

Since $\lambda$ is decreasing, $\left\{x:(z, x) \in \Delta_{j}\right\}$ is a line segment for each $z \in \mathbb{R}$ and $1 \leq j \leq 3$ and, in fact,

$$
\begin{gathered}
\Delta_{1}=\left\{(z, x) \in \Delta: \frac{P^{\prime}(z)}{P(z)} \geq 0, \text { or } \frac{P^{\prime}(z)}{P(z)}<0 \text { and } x \geq \beta_{+}(z)\right\}, \\
\Delta_{2}=\left\{(z, x) \in \Delta: \frac{P^{\prime}(z)}{P(z)}<0 \text { and } x \leq \beta_{-}(z)\right\} \\
\Delta_{3}=\left\{(z, x) \in \Delta: \frac{P^{\prime}(z)}{P(z)}<0 \text { and } \beta_{-}(z) \leq x \leq \beta_{+}(z)\right\} .
\end{gathered}
$$

Lemma 12. On $\Delta_{1}$ and $\Delta_{2}$,

$$
\left|\psi_{3}(x, y, z)\right| \geq c\left\{\begin{array}{l}
\operatorname{dist}((z, x), F)|P(z)| \gamma^{\prime}(z-x) \\
\operatorname{dist}((z, x), F)\left|P^{\prime}(z)\right| \gamma(z-x)
\end{array}\right.
$$

and on $\Delta_{3}$,

$$
\left|\psi_{3}(x, y, z)\right| \geq c\left\{\begin{array}{l}
\operatorname{dist}((z, x), F)^{2}|P(z)| \gamma^{\prime}(z-x), \\
\operatorname{dist}((z, x), F)^{2}\left|P^{\prime}(z)\right| \gamma(z-x) .
\end{array}\right.
$$

Before we begin the proof of Lemma 12, we remind the reader that

$$
\alpha_{y}(z) \leq \beta(z) \leq y
$$

and

$$
\beta_{-}(z) \leq \beta(z) \leq \beta_{+}(z)
$$

on $\Delta_{2} \cup \Delta_{3}$.

\section{Proof. Considering $\Delta_{1}$ :}

If $\frac{P^{\prime}(z)}{P(z)} \geq 0$, then by Lemma 5

$$
\left|\psi_{3}(x, y, z)\right| \geq c\left\{\begin{array}{l}
|x-y \| P(z)| \gamma^{\prime}(z-x), \\
|x-y|\left|P^{\prime}(z)\right| \gamma(z-x),
\end{array}\right.
$$

which implies (13) since $|x-y| \geq \operatorname{dist}((z, x), F)$. We will use Lemma[5]in this way several times in subsequent estimates. 
If $-\frac{1}{2} \frac{\gamma^{\prime \prime}(z-x)}{\gamma^{\prime}(z-x)} \leq \frac{P^{\prime}(z)}{P(z)}<0$, then by the Generalised Mean Value Theorem,

$$
\begin{aligned}
\left|\frac{P^{\prime}(z)(\gamma(z-x)-\gamma(z-y))}{P(z)\left(\gamma^{\prime}(z-x)-\gamma^{\prime}(z-y)\right)}\right| & =\left|\frac{P^{\prime}(z)}{P(z)}\right| \frac{\gamma^{\prime}(\theta)}{\gamma^{\prime \prime}(\theta)} \\
& (\text { for some } z-y<\theta<z-x) \\
\leq & \left|\frac{P^{\prime}(z)}{P(z)}\right| \frac{\gamma^{\prime}(z-x)}{\gamma^{\prime \prime}(z-x)} \leq \frac{1}{2},
\end{aligned}
$$

since $\lambda$ is decreasing. (13) now follows on $\Delta_{1}$.

Considering $\Delta_{2}$ :

$$
\psi_{31}(x, y, z)=-P(z) \gamma^{\prime \prime}(z-x)-P^{\prime}(z) \gamma^{\prime}(z-x) .
$$

On $\Delta_{2},\left|\frac{P^{\prime}(z)}{P(z)}\right|>2 \frac{\gamma^{\prime \prime}(z-x)}{\gamma^{\prime}(z-x)}$, and so

$$
\left|\frac{P^{\prime}(z) \gamma^{\prime}(z-x)}{P(z) \gamma^{\prime \prime}(z-x)}\right| \geq 2 .
$$

Consequently, $\psi_{31}$ is of constant sign as a function of $x$ on $\Delta_{2}$, and so by (14),

$$
\left|\psi_{31}(x, y, z)\right| \geq c\left\{\begin{array}{l}
|P(z)| \gamma^{\prime \prime}(z-x), \\
\left|P^{\prime}(z)\right| \gamma^{\prime}(z-x),
\end{array}\right.
$$

on $\Delta_{2}$. If $\alpha_{y}(z)<\beta_{-}(z)$, then by (15),

$$
\begin{aligned}
\left|\psi_{3}(x, y, z)\right| & \geq c|P(z)|\left|\int_{x}^{\alpha_{y}(z)} \gamma^{\prime \prime}(z-s) d s\right| \\
& =c|P(z)|\left|\gamma^{\prime}(z-x)-\gamma^{\prime}\left(z-\alpha_{y}(z)\right)\right| \\
& \geq c^{\prime}|P(z)|\left|x-\alpha_{y}(z)\right| \gamma^{\prime}(z-x) \\
& \geq c^{\prime} \operatorname{dist}((z, x), F)|P(z)| \gamma^{\prime}(z-x) .
\end{aligned}
$$

On the other hand, by (15),

$$
\begin{aligned}
\left|\psi_{3}(x, y, z)\right| & \geq c\left|P^{\prime}(z)\right|\left|\int_{x}^{\alpha_{y}(z)} \gamma^{\prime}(z-s) d s\right| \\
& =c\left|P^{\prime}(z)\right|\left|\gamma(z-x)-\gamma\left(z-\alpha_{y}(z)\right)\right| \\
& \geq c^{\prime}\left|P^{\prime}(z)\right|\left|x-\alpha_{y}(z)\right| \gamma(z-x) \\
& \geq c^{\prime} \operatorname{dist}((z, x), F)\left|P^{\prime}(z)\right| \gamma(z-x) .
\end{aligned}
$$

If $\alpha_{y}(z) \geq \beta_{-}(z)$, then

$$
\begin{aligned}
\left|\psi_{3}(x, y, z)\right| & \geq c|P(z)|\left|\int_{x}^{\beta_{-}(z)} \gamma^{\prime \prime}(z-s) d s\right| \\
& =c|P(z)|\left|\gamma^{\prime}(z-x)-\gamma^{\prime}\left(z-\beta_{-}(z)\right)\right| \\
& \geq c^{\prime}|P(z)|\left|x-\beta_{-}(z)\right| \gamma^{\prime}(z-x) \\
& \geq c^{\prime} \operatorname{dist}((z, x), F)|P(z)| \gamma^{\prime}(z-x)
\end{aligned}
$$

and

$$
\left|\psi_{3}(x, y, z)\right| \geq c^{\prime} \operatorname{dist}((z, x), F)\left|P^{\prime}(z)\right| \gamma(z-x) .
$$




\section{Considering $\Delta_{3}$ :}

$$
\begin{aligned}
\frac{\partial}{\partial x}\left(\frac{\psi_{31}(x, y, z)}{\gamma^{\prime}(z-x)}\right) & =-P(z) \frac{\partial}{\partial x}\left(\frac{\lambda(z-x)}{z-x}\right) \\
& =-P(z)\left(\frac{\lambda^{\prime}(z-x)}{z-x}-\frac{\lambda(z-x)}{(z-x)^{2}}\right) .
\end{aligned}
$$

Since $\lambda$ is decreasing, $\frac{\psi_{31}(x, y, z)}{\gamma^{\prime}(z-x)}$ is a monotone function of $x$, and since $1 \leq z-x \leq 2$,

$$
\left|\frac{\partial}{\partial x}\left(\frac{\psi_{31}(x, y, z)}{\gamma^{\prime}(z-x)}\right)\right| \geq \frac{1}{2} \frac{\gamma^{\prime \prime}(z-x)}{\gamma^{\prime}(z-x)}|P(z)| .
$$

On $\Delta_{3}$,

$$
|P(z)| \gamma^{\prime \prime}(z-x) \sim\left|P^{\prime}(z)\right| \gamma^{\prime}(z-x)
$$

and so

$$
\left|\frac{\partial}{\partial x}\left(\frac{\psi_{31}(x, y, z)}{\gamma^{\prime}(z-x)}\right)\right| \geq c\left|P^{\prime}(z)\right| .
$$

Since $\frac{\psi_{31}(x, y, z)}{\gamma^{\prime}(z-x)}$ is monotone as a function of $x$, and $(z, \beta(z)) \in \Delta_{3}$, (18) implies

$$
\left|\frac{\psi_{31}(x, y, z)}{\gamma^{\prime}(z-x)}\right| \geq c\left|P^{\prime}(z)\right||x-\beta(z)|,
$$

which implies, by (17),

$$
\left|\psi_{31}(x, y, z)\right| \geq c\left\{\begin{array}{l}
\left|P^{\prime}(z)\right||x-\beta(z)| \gamma^{\prime}(z-x), \\
|P(z)||x-\beta(z)| \gamma^{\prime \prime}(z-x) .
\end{array}\right.
$$

The estimates we make now will depend on the location of $x$ relative to the zeros of $\psi_{3}$, i.e., $\alpha_{y}(z)$ and $y$. Since $\alpha_{y}(z) \leq \beta(z) \leq y$ we consider three cases:

(i) $x \leq \alpha_{y}(z)$,

(ii) $\alpha_{y}(z)<x \leq \beta(z)$,

(iii) $x \geq \beta(z)$.

Since $\gamma \in C^{3}(\mathbb{R}), \psi_{31} \in C^{1}(\mathbb{R})$ as a function of $x$. Consequently, $\psi_{31}$ is of constant sign in each of the regions (i)-(iii). This observation allows us to make the following estimates.

In case (i), we may suppose that $\alpha_{y}(z) \geq \beta_{-}(z)$ (or else (i) is vacuous), and so

$$
\begin{aligned}
\left|\psi_{3}(x, y, z)\right| & \geq C|P(z)| \int_{x}^{\alpha_{y}(z)}|t-\beta(z)| \gamma^{\prime \prime}(z-t) d t \\
& \geq C|P(z)| \int_{x}^{\frac{\alpha_{y}(z)+x}{2}}|t-\beta(z)| \gamma^{\prime \prime}(z-t) d t \\
& \geq C|P(z)|\left|\frac{\alpha_{y}(z)+x}{2}-\beta(z)\right|\left|\gamma^{\prime}\left(z-\frac{\alpha_{y}(z)+x}{2}\right)-\gamma^{\prime}(z-x)\right| \\
& \geq C|P(z)| \frac{\alpha_{y}(z)+x}{2}-\alpha_{y}(z)|| \frac{\alpha_{y}(z)+x}{2}-x \mid \gamma^{\prime}(z-x) \\
& =C^{\prime}|P(z)|\left|x-\alpha_{y}(z)\right|^{2} \gamma^{\prime}(z-x) .
\end{aligned}
$$

Similarly,

$$
\left|\psi_{3}(x, y, z)\right| \geq C\left|P^{\prime}(z)\right|\left|x-\alpha_{y}(z)\right|^{2} \gamma(z-x) .
$$


In case (ii), if $\alpha_{y}(z) \geq \beta_{-}(z)$, then

$$
\begin{aligned}
\left|\psi_{3}(x, y, z)\right| & \geq C|P(z)| \int_{\alpha_{y}(z)}^{x}|t-\beta(z)| \gamma^{\prime \prime}(z-t) d t \\
& \geq C|P(z)||x-\beta(z)|\left|\gamma^{\prime}(z-x)-\gamma^{\prime}\left(z-\alpha_{y}(z)\right)\right| \\
& \geq C|P(z)||x-\beta(z)|\left|x-\alpha_{y}(z)\right| \gamma^{\prime}(z-x) .
\end{aligned}
$$

Similarly,

$$
\left|\psi_{3}(x, y, z)\right| \geq C\left|P^{\prime}(z)\right||x-\beta(z)|\left|x-\alpha_{y}(z)\right| \gamma(z-x) .
$$

If $\alpha_{y}(z)<\beta_{-}(z)$, we observe that $\beta_{-}(z)<x \leq \beta(z)$, and

$$
\begin{aligned}
\left|\psi_{3}(x, y, z)\right| & \geq C|P(z)| \int_{\beta_{-}(z)}^{x}|t-\beta(z)| \gamma^{\prime \prime}(z-t) d t \\
& \geq C|P(z)||x-\beta(z)|\left|\gamma^{\prime}(z-x)-\gamma^{\prime}\left(z-\beta_{-}(z)\right)\right| \\
& \geq C|P(z)||x-\beta(z)|\left|x-\beta_{-}(z)\right| \gamma^{\prime}(z-x) .
\end{aligned}
$$

Similarly,

$$
\left|\psi_{3}(x, y, z)\right| \geq C\left|P^{\prime}(z)\right||x-\beta(z)|\left|x-\beta_{-}(z)\right| \gamma(z-x) .
$$

In case (iii), if $y \leq \beta_{+}(z)$, then

$$
\begin{aligned}
\left|\psi_{3}(x, y, z)\right| & \geq C|P(z)| \int_{x}^{y}|t-\beta(z)| \gamma^{\prime \prime}(z-t) d t \\
& \geq C|P(z) \| x-\beta(z)|\left|\gamma^{\prime}(z-x)-\gamma^{\prime}(z-y)\right| \\
& \geq C|P(z) \| x-\beta(z)||x-y| \gamma^{\prime}(z-x) .
\end{aligned}
$$

Similarly,

$$
\left|\psi_{3}(x, y, z)\right| \geq C\left|P^{\prime}(z)\right||x-\beta(z)||x-y| \gamma(z-x) .
$$

If $y>\beta_{+}(z)$, then we observe that $\beta(z) \leq x \leq \beta_{+}(z)$ and argue as before.

Lemma 13. Let $P$ be a real monic polynomial of degree $n$ and of one real variable. Let $U$ be the union of the set of roots of $P$ and of $P^{\prime}$ over $\mathbb{R}$. There exists $C>0$, depending only on $n$, such that if $\operatorname{dist}(x, U)>\epsilon$, then

$$
|P(x)| \geq C \epsilon^{n},
$$

for all $\epsilon>0$.

Proof. Let $\epsilon>0$, and suppose $x$ is chosen so that $\operatorname{dist}(x, U)>\epsilon$. Let $y_{x} \in U$ be such that $\left|x-y_{x}\right|$ is minimal; so $\left|x-y_{x}\right|>\epsilon$. Without loss of generality, we may suppose that $y_{x}>x$. We observe that $P$ is monotone on $\left[x, y_{x}\right]$. There are two cases to consider.

Case 1: $P\left(y_{x}\right)=0$

By Lemma 6 .

$$
|\{z \in \mathbb{R}:|P(z)|<|P(x)|\}| \leq c|P(x)|^{\frac{1}{n}},
$$

with constant $c$ independent of the coefficients of $P$. Since $P$ is monotone on $\left[x, y_{x}\right]$, (19) implies that

$$
|P(x)| \geq c^{\prime}\left|x-y_{x}\right|^{n} \geq c^{\prime} \epsilon^{n},
$$

as required.

Case 2: $P\left(y_{x}\right) \neq 0$ 
Since $y_{x} \in U, P^{\prime}\left(y_{x}\right)=0$. Let $y_{x}^{\prime} \in U$ be such that $y_{x}^{\prime}<x<y_{x}$ and is maximal in $U$.

If $P\left(y_{x}^{\prime}\right)=0$, the argument in case 1 applies.

If $P\left(y_{x}^{\prime}\right) \neq 0$, then since $y_{x}^{\prime}$ was chosen maximally, $P$ is single-signed on $\left[y_{x}^{\prime}, y_{x}\right]$. Without loss of generality, we may suppose that $\left|P\left(y_{x}\right)\right|<\left|P\left(y_{x}^{\prime}\right)\right|$, and hence $|P(x)| \geq\left|P(x)-P\left(y_{x}\right)\right|$. An application of the argument in case 1 to the polynomial $Q(z)=P(z)-P\left(y_{x}\right)$ completes the proof of Lemma 13 .

Remark. By applying Lemma 13] to the estimates in the statement of Lemma 12] we can conclude that on $\Delta$,

$$
\left|\psi_{3}(x, y, z)\right| \geq C\left\{\begin{array}{l}
\operatorname{dist}((z, x), F)^{n+2} \gamma^{\prime}(z-x) \\
\operatorname{dist}((z, x), F)^{n+1} \gamma(z-x)
\end{array}\right.
$$

We now show how Lemmas 12 and 13 finish the proof of Lemma 8 .

Integrating by parts,

$$
\begin{aligned}
\mathbb{L}_{l, m}(x, y)= & \int_{\left\{z:(z, x) \in B_{l, m}\right\}} e^{i \mu \psi(x, y, z)} \frac{d z}{(z-x)(z-y)} \\
= & \frac{1}{i \mu} \int_{\left\{z:(z, x) \in B_{l, m}\right\}} \frac{1}{\psi_{3}(x, y, z)} \frac{1}{(z-x)(z-y)} \frac{\partial}{\partial z}\left(e^{i \mu \psi(x, y, z)}\right) d z \\
= & \frac{1}{i \mu}\left[\frac{e^{i \mu \psi(x, y, z)}}{\psi_{3}(x, y, z)(z-x)(z-y)}\right]_{\left\{z:(z, x) \in \partial B_{l, m}\right\}} \\
& -\frac{1}{i \mu} \int_{\left\{z:(z, x) \in B_{l, m}\right\}} \frac{\partial}{\partial z}\left(\frac{1}{\psi_{3}(x, y, z)} \frac{1}{(z-x)(z-y)}\right) e^{i \mu \psi(x, y, z)} d z
\end{aligned}
$$

and so, for $y$ fixed,

$$
\begin{aligned}
\int\left|\mathbb{L}_{l, m}(x, y)\right| d x & \leq \frac{1}{\mu} \int_{\partial B_{l, m}}\left|\frac{1}{\psi_{3}(x, y, z)(z-x)(z-y)}\right| d \sigma_{l, m} \\
& +\frac{1}{\mu} \int_{B_{l, m}}\left|\frac{\partial}{\partial z}\left(\frac{1}{\psi_{3}(x, y, z)} \frac{1}{(z-x)(z-y)}\right)\right| d z d x
\end{aligned}
$$

where $d \sigma_{l, m}$ is Lebesgue measure on $\partial B_{l, m}$.

By (20), the partially integrated term in (21) is bounded above by

$$
\frac{1}{\mu} \int_{\partial B_{l, m}}\left|\frac{1}{\psi_{3}(x, y, z)}\right| d \sigma_{l, m} \leq \frac{C}{\mu}\left|\partial B_{l, m}\right| \frac{1}{2^{-(n+1) l}} \leq \frac{C 2^{n l}}{\mu},
$$

uniformly in $y$.

The remaining term in (21) is bounded above by

$$
\begin{aligned}
\frac{1}{\mu} \int_{B_{l, m}} & \left|\frac{\psi_{33}(x, y, z)}{\psi_{3}(x, y, z)^{2}}\right| d z d x \\
& +\frac{1}{\mu} \int_{B_{l, m}}\left|\frac{\partial}{\partial z}\left(\frac{1}{(z-x)(z-y)}\right)\right| d z d x \sup _{(z, x) \in B_{l, m}}\left|\frac{1}{\psi_{3}(x, y, z)}\right| .
\end{aligned}
$$

The second term in (23) is (by (201) ) bounded above by $\frac{C 2^{n l}}{\mu}$. 
The remaining term in (23) is, by the triangle inequality, bounded by

$$
\begin{aligned}
& \frac{1}{\mu} \int_{B_{l, m}} \frac{\left|P(z)\left(\gamma^{\prime \prime}(z-x)-\gamma^{\prime \prime}(z-y)\right)\right|}{\psi_{3}(x, y, z)^{2}} d z d x \\
& +\frac{1}{\mu} \int_{B_{l, m}} \frac{\left|P^{\prime}(z)\left(\gamma^{\prime}(z-x)-\gamma^{\prime}(z-y)\right)\right|}{\psi_{3}(x, y, z)^{2}} d z d x \\
& +\frac{2}{\mu} \int_{B_{l, m}} \frac{\left|P^{\prime \prime}(z)(\gamma(z-x)-\gamma(z-y))\right|}{\psi_{3}(x, y, z)^{2}} d z d x \\
& =I+I I+I I I .
\end{aligned}
$$

By Lemmas 12 and 13 ,

$$
\begin{aligned}
I & \leq \frac{C}{\mu} \int_{B_{l, m}} \frac{\left|P(z) \| \gamma^{\prime \prime}(z-x)-\gamma^{\prime \prime}(z-y)\right|}{P(z)^{2} 2^{-4 l} \gamma^{\prime}(z-x)^{2}} d z d x \\
& \leq \frac{C 2^{4 l}}{\mu} \int_{B_{l, m}} \frac{1}{|P(z)|}\left(\frac{\gamma^{\prime \prime}(z-x)}{\gamma^{\prime}(z-x)^{2}}+\frac{\gamma^{\prime \prime}(z-y)}{\gamma^{\prime}(z-y)^{2}}\right) d z d x \\
& \leq \frac{C 2^{(n+4) l}}{\mu}\left|\int_{B_{l, m}} \frac{\partial}{\partial z}\left(\frac{1}{\gamma^{\prime}(z-x)}+\frac{1}{\gamma^{\prime}(z-y)}\right) d z d x\right| \\
& \leq \frac{C 2^{(n+3) l}}{\mu}
\end{aligned}
$$

uniformly in $y$.

Again by Lemmas 12 and 13

$$
\begin{aligned}
I I & \leq \frac{C}{\mu} \int_{B_{l, m}} \frac{\left|P^{\prime}(z)\right|\left|\gamma^{\prime}(z-x)-\gamma^{\prime}(z-y)\right|}{P(z)^{2} 2^{-4 l} \gamma^{\prime}(z-x)^{2}} d z d x \\
& \leq \frac{C 2^{4 l}}{\mu} \int_{B_{l, m}}\left|\frac{\partial}{\partial z}\left(\frac{1}{P(z)}\right)\right| d z d x \\
& \leq \frac{C 2^{4 l}}{\mu}\left|\int_{B_{l, m}} \frac{\partial}{\partial z}\left(\frac{1}{P(z)}\right) d z d x\right| \\
& \left(\text { since } P \text { is monotone on } B_{l, m}\right) \\
& \leq \frac{C 2^{(n+3) l}}{\mu}
\end{aligned}
$$

uniformly in $y$.

Similarly,

$$
\begin{aligned}
I I I & \leq \frac{C}{\mu} \int_{B_{l, m}} \frac{\left|P^{\prime \prime}(z)\right||\gamma(z-x)-\gamma(z-y)|}{P^{\prime}(z)^{2} 2^{-4 l} \gamma(z-x)^{2}} d z d x \\
& \leq \frac{C 2^{4 l}}{\mu} \int_{B_{l, m}}\left|\frac{\partial}{\partial z}\left(\frac{1}{P^{\prime}(z)}\right)\right| d z d x \\
& \leq \frac{C 2^{4 l}}{\mu}\left|\int_{B_{l, m}} \frac{\partial}{\partial z}\left(\frac{1}{P^{\prime}(z)}\right) d z d x\right| \\
& \left(\text { since } P^{\prime} \text { is monotone on } B_{l, m}\right) \\
& \leq \frac{C 2^{(n+2) l}}{\mu}
\end{aligned}
$$


uniformly in $y$.

Combining these estimates gives

$$
\sup _{y} \int\left|\mathbb{L}_{l, m}(x, y)\right| d x \leq \frac{C 2^{(n+3) l}}{\mu}
$$

which is (12) with $M=n+3$.

This concludes the proof of Lemma 8 .

Proof of Lemma 9. Suppose the roots of $P$ are $\left\{\nu_{j}\right\}_{j=1}^{m} \subset \mathbb{R}$ and $\left\{\beta_{j}\right\}_{j=1}^{n^{\prime}},\left\{\bar{\beta}_{j}\right\}_{j=1}^{n^{\prime}}$ $\subset \mathbb{C} \backslash \mathbb{R}$, where $n^{\prime}=\frac{n-m}{2}$ and $\beta_{j}=a_{j}+i b_{j}$. Now,

$$
\widetilde{P}_{k}(x)=\Pi_{j=1}^{m}\left(x-2^{-k} \nu_{j}\right) \Pi_{j=1}^{n^{\prime}}\left(x-2^{-k} \beta_{j}\right)\left(x-2^{-k} \bar{\beta}_{j}\right),
$$

and so,

$$
\begin{aligned}
\frac{\widetilde{P}_{k}^{\prime}(x)}{\widetilde{P}_{k}(x)} & =\sum_{j=1}^{m} \frac{1}{\left(x-2^{-k} \nu_{j}\right)}+\sum_{j=1}^{n^{\prime}} \frac{1}{\left(x-2^{-k} \beta_{j}\right)}+\frac{1}{\left(x-2^{-k} \bar{\beta}_{j}\right)} \\
& =\sum_{j=1}^{m} \frac{1}{\left(x-2^{-k} \nu_{j}\right)}+2 \sum_{j=1}^{n^{\prime}} \operatorname{Re}\left(\frac{1}{x-2^{-k} \beta_{j}}\right) \\
& =\sum_{j=1}^{m} \frac{1}{\left(x-2^{-k} \nu_{j}\right)}+2 \sum_{j=1}^{n^{\prime}} \frac{x-2^{-k} a_{j}}{\left(x-2^{-k} a_{j}\right)^{2}+\left(2^{-k} b_{j}\right)^{2}}
\end{aligned}
$$

and

$$
\begin{aligned}
\left(\frac{\widetilde{P}_{k}^{\prime}}{\widetilde{P}_{k}}\right)^{\prime}(x) & =-\sum_{j=1}^{m} \frac{1}{\left(x-2^{-k} \nu_{j}\right)^{2}}-2 \sum_{j=1}^{n^{\prime}} \operatorname{Re}\left(\frac{1}{\left(x-2^{-k} \beta_{j}\right)^{2}}\right) \\
& =-\sum_{j=1}^{m} \frac{1}{\left(x-2^{-k} \nu_{j}\right)^{2}}-2 \sum_{j=1}^{n^{\prime}} \frac{\left(x-2^{-k} a_{j}\right)^{2}-\left(2^{-k} b_{j}\right)^{2}}{\left(\left(x-2^{-k} a_{j}\right)^{2}+\left(2^{-k} b_{j}\right)^{2}\right)^{2}}
\end{aligned}
$$

If $x \in E_{k}$, then, by definition,

$$
\sum_{j=1}^{m} \frac{1}{\left(x-2^{-k} \nu_{j}\right)^{2}}+2 \sum_{j=1}^{n^{\prime}} \frac{\left(x-2^{-k} a_{j}\right)^{2}-\left(2^{-k} b_{j}\right)^{2}}{\left(\left(x-2^{-k} a_{j}\right)^{2}+\left(2^{-k} b_{j}\right)^{2}\right)^{2}}<0
$$

and

$$
\left|\sum_{j=1}^{m} \frac{1}{\left(x-2^{-k} \nu_{j}\right)}+2 \sum_{j=1}^{n^{\prime}} \frac{x-2^{-k} a_{j}}{\left(x-2^{-k} a_{j}\right)^{2}+\left(2^{-k} b_{j}\right)^{2}}\right| \geq \frac{\lambda_{0}}{4} .
$$

By the triangle inequality, (28) implies that

$$
\sum_{j=1}^{m} \frac{1}{\left|x-2^{-k} \nu_{j}\right|}+2 \sum_{j=1}^{n^{\prime}} \frac{\left|x-2^{-k} a_{j}\right|}{\left(x-2^{-k} a_{j}\right)^{2}+\left(2^{-k} b_{j}\right)^{2}} \geq \frac{\lambda_{0}}{4},
$$

and by the equivalence of the $l^{1}$ and $l^{2}$ norms on $\mathbb{R}^{n}$, the above implies

$$
\sum_{j=1}^{m} \frac{1}{\left(x-2^{-k} \nu_{j}\right)^{2}}+2 \sum_{j=1}^{n^{\prime}} \frac{\left(x-2^{-k} a_{j}\right)^{2}}{\left(\left(x-2^{-k} a_{j}\right)^{2}+\left(2^{-k} b_{j}\right)^{2}\right)^{2}} \geq c \lambda_{0}^{2},
$$


for some constant $c$ depending only on $n$. Combining (27) and (29), we obtain

$$
\sum_{j=1}^{n^{\prime}} \frac{\left(2^{-k} b_{j}\right)^{2}}{\left(\left(x-2^{-k} a_{j}\right)^{2}+\left(2^{-k} b_{j}\right)^{2}\right)^{2}} \geq c \lambda_{0}^{2},
$$

or, using the $l^{1}$ norm,

$$
\sum_{j=1}^{n^{\prime}} \frac{\left|2^{-k} b_{j}\right|}{\left(x-2^{-k} a_{j}\right)^{2}+\left(2^{-k} b_{j}\right)^{2}} \geq c^{\prime} \lambda_{0} .
$$

If $x$ satisfies (31), then

$$
\frac{\left|2^{-k} b_{j}\right|}{\left(x-2^{-k} a_{j}\right)^{2}+\left(2^{-k} b_{j}\right)^{2}} \geq \frac{c^{\prime} \lambda_{0}}{n^{\prime}}
$$

for some $1 \leq j \leq n^{\prime}$. Hence,

$$
E_{k} \subset \bigcup_{j=1}^{n^{\prime}} E_{j k}
$$

where

$$
E_{j k}=\left\{x \in \mathbb{R}:\left|2^{-k} b_{j}\right| \leq \lambda_{0}^{-1} \text { and }\left|x-2^{-k} a_{j}\right| \leq \lambda_{0}^{-1 / 2}\left|2^{-k} b_{j}\right|^{1 / 2}\right\},
$$

and so, for $0<\alpha \leq 1$,

$$
\sum_{k \geq 0}\left|E_{k}\right|^{\alpha} \leq \sum_{k \geq 0}\left(\sum_{j=1}^{n^{\prime}}\left|E_{j k}\right|\right)^{\alpha} \leq \sum_{j=1}^{n^{\prime}} \sum_{k \geq 0}\left|E_{j k}\right|^{\alpha} .
$$

For $\alpha>1$, the above holds with a constant factor depending only on $n^{\prime}$ and $\alpha$.

Since $E_{j k}=\emptyset$ if $\left|2^{-k} b_{j}\right|>\lambda_{0}^{-1}$ and $\left|E_{j k}\right| \leq \lambda_{0}^{-1 / 2}\left|2^{-k} b_{j}\right|^{1 / 2}$ if $\left|2^{-k} b_{j}\right| \leq \lambda_{0}^{-1}$,

$$
\sum_{k \geq 0}\left|E_{j k}\right|^{\alpha} \leq C \lambda_{0}^{-\alpha}
$$

uniformly in $j$, for some constant $C$ depending on $\alpha$. Consequently,

$$
\sum_{k \geq 0}\left|E_{k}\right|^{\alpha} \leq C n^{\prime} \lambda_{0}^{-\alpha}
$$

This completes the proof of Lemma 9, and hence the inductive step, which leads to Theorem 1

\section{The proof of Theorem 2}

Let $\nu \in C_{c}^{\infty}(\mathbb{R})$ be such that $\nu \geq 0$ and $\int \nu=1$. For $k \in \mathbb{Z}$, let

$$
\nu_{k}(x)=2^{-k} \nu\left(2^{-k} x\right) \text {. }
$$

For $f \in L^{2}\left(\mathbb{R}^{2}\right)$, let

$$
\mathcal{M}_{k} f\left(x_{1}, x_{2}\right)=\frac{1}{2^{k}} \int_{2^{k}}^{2^{k+1}} f\left(x_{1}-t, x_{2}-P\left(x_{1}\right) \gamma(t)\right) d t
$$

and

$$
\begin{aligned}
& \mathcal{N}_{k} f\left(x_{1}, x_{2}\right) \\
& \quad=\frac{1}{2^{k} \gamma\left(2^{k}\right)\left|P\left(x_{1}\right)\right|} \int_{\mathbb{R}} \int_{\mathbb{R}} \nu\left(\frac{x_{1}-y_{1}}{2^{k}}\right) \nu\left(\frac{x_{2}-y_{2}}{\gamma\left(2^{k}\right) P\left(x_{1}\right)}\right) f\left(y_{1}, y_{2}\right) d y_{1} d y_{2} .
\end{aligned}
$$


For a polynomial $P$, which we may take to be monic, we make the majorisation

$$
\mathcal{M} f(x) \lesssim \sup _{k}\left|\mathcal{M}_{k} f(x)\right| \leq \mathcal{G} f(x)+\mathcal{N} f(x),
$$

where $\mathcal{N} f(x)=\sup _{k}\left|\mathcal{N}_{k} f(x)\right|$ and

$$
\mathcal{G} f(x)=\left(\sum_{k \in \mathbb{Z}}\left|\left(\mathcal{M}_{k}-\mathcal{N}_{k}\right) f(x)\right|^{2}\right)^{1 / 2} .
$$

For each $x \in \mathbb{R}^{2}$,

$$
\mathcal{N} f(x) \lesssim M_{2}\left(M_{1}(f)\right)(x)
$$

where $M_{1}$ and $M_{2}$ are the Hardy-Littlewood maximal functions in the first and second coordinate directions respectively. Hence $\mathcal{N}$ is bounded on $L^{2}\left(\mathbb{R}^{2}\right)$ uniformly in the coefficients of $P$. Consequently, it suffices to show that $\mathcal{G}$ is bounded on $L^{2}\left(\mathbb{R}^{2}\right)$ uniformly in the coefficients of $P$. This in turn would follow from the boundedness of

$$
\mathcal{L}=\sum_{k} \epsilon_{k}\left(\mathcal{M}_{k}-\mathcal{N}_{k}\right)
$$

for $\epsilon_{k} \in\{-1,1\}$, uniformly in the choice of $\left\{\epsilon_{k}\right\}$ and the coefficients of $P$.

The uniform boundedness of the operator $\mathcal{L}$ will be obtained by induction on the degree of $P$.

The inductive hypothesis. Suppose $\mathcal{L}$ is bounded on $L^{2}\left(\mathbb{R}^{2}\right)$ for all polynomials of degree less than $n$ uniformly in the choice of $\left\{\epsilon_{k}\right\}$ and the coefficients of $P$.

The case $n=0$. In this case, the operator $\mathcal{L}$ is translation invariant and can be handled using the Fourier transform. This is essentially done in [6].

- Let $P$ be a real-valued polynomial of degree $n$, and let $\left\{\epsilon_{k}\right\}$ be an arbitrary sequence of 1's and -1 's. By taking the Fourier transform in the second variable, it suffices to bound

$$
(\mathcal{L})_{\lambda}=\sum_{k} \epsilon_{k}\left(\left(\mathcal{M}_{k}\right)_{\lambda}-\left(\mathcal{N}_{k}\right)_{\lambda}\right)
$$

on $L^{2}(\mathbb{R})$ uniformly in $\lambda$, where

$$
\left(\mathcal{M}_{k}\right)_{\lambda} f(x)=\frac{1}{2^{k}} \int_{2^{k}}^{2^{k+1}} e^{i \lambda P(x) \gamma(y)} f(x-y) d y
$$

and

$$
\left(\mathcal{N}_{k}\right)_{\lambda} f(x)=\widehat{\nu}\left(\lambda \gamma\left(2^{k}\right) P(x)\right) \nu_{k} * f(x) .
$$

As in the proof of Theorem 1 by rescaling it suffices to consider the case where $\lambda=1, P$ is monic, and $\gamma(1)=1$. For ease of notation we set $M_{k}=\left(\mathcal{M}_{k}\right)_{1}$, $N_{k}=\left(\mathcal{N}_{k}\right)_{1}$, and $L=(\mathcal{L})_{1}$.

We first turn to the local part,

$$
L_{\mathrm{loc}}=\sum_{k \leq 0} \epsilon_{k}\left(M_{k}-N_{k}\right)
$$


It suffices to consider $L_{\mathrm{loc}}$ acting on functions supported in balls of radius 1 . Let $f \in L^{2}(\mathbb{R})$ be supported in $B(b ; 1)$ for some $b \in \mathbb{R}$, and let $Q_{b}(x)=P(x)-(x-b)^{n}$. Let

$$
L_{\mathrm{loc}}^{\prime}=\sum_{k \leq 0} \epsilon_{k}\left(M_{k}^{\prime}-N_{k}^{\prime}\right)
$$

where

$$
M_{k}^{\prime} f(x)=\frac{1}{2^{k}} \int_{2^{k}}^{2^{k+1}} e^{i Q_{b}(x) \gamma(y)} f(x-y) d y
$$

and

$$
N_{k}^{\prime} f(x)=\widehat{\nu}\left(\gamma\left(2^{k}\right) Q_{b}(x)\right) \nu_{k} * f(x) .
$$

Since $Q_{b}$ is a polynomial of degree less than $n$, by the inductive hypothesis, $L_{\text {loc }}^{\prime}$ is bounded on $L^{2}(\mathbb{R})$ uniformly in $\left\{\epsilon_{k}\right\}, b$, and the coefficients of $P$. Now,

$$
\begin{aligned}
\left|\left(L_{\mathrm{loc}}-L_{\mathrm{loc}}^{\prime}\right) f(x)\right| & \leq\left|\sum_{k \leq 0} \epsilon_{k}\left(M_{k}-M_{k}^{\prime}\right) f(x)\right|+\left|\sum_{k \leq 0} \epsilon_{k}\left(N_{k}-N_{k}^{\prime}\right) f(x)\right| \\
& =|I f(x)|+|I I f(x)| .
\end{aligned}
$$

Now,

$$
\begin{aligned}
|I f(x)| & \leq \sum_{k \leq 0} \frac{1}{2^{k}} \int_{2^{k}}^{2^{k+1}}\left|e^{i P(x) \gamma(y)}-e^{i Q_{b}(x) \gamma(y)}\right||f(x-y)| d y \\
& \leq \sum_{k \leq 0} \frac{1}{2^{k}} \int_{2^{k}}^{2^{k+1}}|x-b|^{n} \gamma(y)|f(x-y)| d y \\
& \lesssim \sum_{k \leq 0} \gamma\left(2^{k+1}\right) \frac{1}{2^{k}} \int_{2^{k}}^{2^{k+1}} \frac{\gamma(y)}{\gamma\left(2^{k+1}\right)}|f(x-y)| d y \\
& \lesssim M f(x) \sum_{k \leq 0} \gamma\left(2^{k+1}\right) \\
& \lesssim M f(x),
\end{aligned}
$$

where $M$ is the Hardy-Littlewood maximal function. Since $M$ is bounded on $L^{2}(\mathbb{R})$, $I$ is uniformly bounded on $L^{2}(\mathbb{R})$.

Now,

$$
\begin{aligned}
|I I f(x)| & \leq \sum_{k \leq 0}\left|\left[\widehat{\nu}\left(\gamma\left(2^{k}\right) P(x)\right)-\widehat{\nu}\left(\gamma\left(2^{k}\right) Q_{b}(x)\right)\right] \nu_{k} * f(x)\right| \\
& \leq|x-b|^{n} \gamma\left(2^{k}\right) \int_{\mathbb{R}}|s \nu(s)| d s\left|\nu_{k} * f(x)\right| \\
& \lesssim M f(x) \sum_{k \leq 0} \gamma\left(2^{k}\right) \\
& \lesssim M f(x),
\end{aligned}
$$

where again $M$ is the Hardy-Littlewood maximal function. Hence $L_{\mathrm{loc}}$ is bounded on $L^{2}(\mathbb{R})$ in the required uniform manner. 
We now turn to the global part,

$$
L_{\text {glob }}=\sum_{k>0} \epsilon_{k}\left(M_{k}-N_{k}\right) .
$$

Clearly it suffices to bound

$$
M_{\mathrm{glob}}=\sum_{k>0} \epsilon_{k} M_{k}
$$

and

$$
N_{\text {glob }}=\sum_{k>0} \epsilon_{k} N_{k}
$$

separately on $L^{2}(\mathbb{R})$. By the triangle inequality,

$$
\left\|M_{\text {glob }}\right\|_{2-2} \leq \sum_{k>0}\left\|M_{k}\right\|_{2-2},
$$

which can be controlled as in the proof of Theorem 1 This estimate represents the majority of our analysis.

In order to control (34) we will make some estimates on the kernel of $N_{k}$, which is given by

$$
N_{k}(x, y)=\nu_{k}(x-y) \widehat{\nu}\left(\gamma\left(2^{k}\right) P(x)\right) .
$$

It is convenient to make the $L^{2}$ operator norm preserving rescaling $\widetilde{N}_{k}(x, y)=$ $2^{k} N_{k}\left(2^{k} x, 2^{k} y\right)$, so that

$$
\widetilde{N}_{k}(x, y)=\nu(x-y) \widehat{\nu}\left(2^{n k} \gamma\left(2^{k}\right) \widetilde{P}_{k}(x)\right),
$$

where as before $\widetilde{P}_{k}(x)=2^{-n k} P\left(2^{k} x\right)$. Since $\nu \in C_{c}^{\infty}(\mathbb{R})$,

$$
\left|\widetilde{N}_{k}(x, y)\right| \lesssim \nu(x-y)\left|2^{n k} \gamma\left(2^{k}\right) \widetilde{P}_{k}(x)\right|^{-1},
$$

and trivially,

$$
\left|\widetilde{N}_{k}(x, y)\right| \lesssim \nu(x-y)
$$

Using Lemma 6, (35) and (36) imply that

$$
\sup _{y} \int_{\mathbb{R}}\left|\widetilde{N}_{k}(x, y)\right| d x \lesssim\left(2^{n k} \gamma\left(2^{k}\right)\right)^{-\frac{1}{n+1}},
$$

and trivially,

$$
\sup _{x} \int_{\mathbb{R}}\left|\widetilde{N}_{k}(x, y)\right| d y \lesssim 1
$$

Interpolating between the above two estimates gives

$$
\left\|\widetilde{N}_{k}\right\|_{2-2} \lesssim\left(2^{n k} \gamma\left(2^{k}\right)\right)^{-\frac{1}{2 n+2}}
$$

which, by the triangle inequality, implies the uniform $L^{2}(\mathbb{R})$ boundedness of $N_{\text {glob }}$.

This completes the proof of Theorem 2.

Remarks. The $L^{p}$ theory for $\mathcal{H}$ and $\mathcal{M}$, for $p \neq 2$, has not yet been established. These operators may be "lifted" to operators acting on functions of many variables, which have enough homogeneity for an associated Littlewood-Paley theory to be developed; as in [5]. However, the techniques used in the proof of Theorem 1 so far appear to be insufficient to complete this scheme. 


\section{REFERENCES}

1. J. M. Bennett, Some oscillatory singular integrals with variable flat phases, and related operators, Ph.D. thesis, Edinburgh University (1998).

2. A. Carbery, M. Christ, J. Vance, S. Wainger, and D. K. Watson, Operators associated to flat plane curves: $L^{p}$ estimates via dilation methods, Duke Mathematical Journal, Vol. 59, No. 3, 1989. MR 91m: 42017

3. A. Carbery and S. Pérez, Maximal functions and Hilbert transforms along variable flat curves, Math. Research Letters 6 (1999), 237-299. MR 2000f:42008

4. A. Carbery, S. Wainger, and J. Wright, Hilbert transforms and maximal functions associated to flat curves on the Heisenberg group, J. Amer. Math. Soc., Volume 8, Number 1, (1995), 141-179. MR 95g:43010

5. A. Carbery, S. Wainger, and J. Wright, Hilbert transforms and maximal functions along variable flat plane curves. J. Fourier Anal. Appl., Kahane Special Issue (1995), 119-139. MR 97b:42030

6. H. Carlsson, M. Christ, A. Córdoba, J. Duoandikoetxea, J. L. Rubio de Francia, J. Vance, S. Wainger, and D. Weinberg, $L^{p}$ estimates for maximal functions and Hilbert transforms along flat convex curves in $\mathbb{R}^{2}$, Bull. Amer. Math. Soc. (N.S.) 14 (1986), 263-267. MR 87f:42044

7. J. Kim, Hilbert transforms and maximal functions along curves in the Heisenberg group, Ph.D. thesis, University of Wisconsin-Madison (1998).

8. D. H. Phong and E. M. Stein, Hilbert integrals, singular integrals and Radon transforms, I, Acta Math. 157 (1986), 99-157. MR 88i:42028a

9. F. Ricci and E. M. Stein, Harmonic analysis on nilpotent groups and singular integrals I. Oscillatory integrals, J. Funct. Anal. 73 (1987), 179-194. MR 88g:42023

10. A. Seeger, $L^{2}$-estimates for a class of singular oscillatory integrals, Math. Research Letters 1 (1994), 65-73. MR 95e:42005

Department of Mathematics and Statistics, JCMB, Kings Buildings, Mayfield Road, EDINBURGH, EH9 3JZ, SCOTLAND 